\title{
Elementos-traço em solo de área de disposição final de resíduos sólidos urbanos
}

Os elementos-traço podem estar presentes no solo tanto por causas naturais quanto por adições antropogênicas, sendo esta última preocupante, visto que eles não podem ser degradados e acumulam no ambiente. Estudos indicam altos teores de elementos-traço em áreas de disposição final de resíduos sólidos urbanos (RSU), porém isso nem sempre ocorre. Assim, este trabalho buscou verificar se a disposição de RSU da cidade de Pontes e Lacerda/MT, alterou a qualidade do solo local, analisando-se os atributos de fertilidade e os teores dos elementos-traço $\mathrm{As}, \mathrm{Ba}, \mathrm{Co}, \mathrm{Cu}, \mathrm{Cr}, \mathrm{Cd}, \mathrm{Hg}, \mathrm{Mo}, \mathrm{Ni}$, $\mathrm{Pb}$, $\mathrm{Sb}$, Se e $\mathrm{Zn}$. Foram realizadas coletas de solo na área ativa de disposição final de RSU e em áreas de vegetação nativa, no entorno da mesma, totalizando 20 amostras na profundidade $0-0,20$ m. Foram verificadas alterações nos atributos químicos, em especial, acidez ativa e potencial, as quais foram menores na área ativa de disposição final de RSU. Já a saturação por bases e os teores de $\mathrm{K}$ foram mais elevados na área ativa de disposição final de RSU. Os teores de As foram mais elevados na área de vegetação nativa. Os teores de $\mathrm{Cd}, \mathrm{Hg}, \mathrm{Sb}$ e As foram elevados, inclusive na área de vegetação nativa, sendo assim, recomenda-se a realização de novos estudos para a confirmação desses resultados. Foram observados teores acima dos VP da Resolução CONAMA 420/2009 para o As, Sb, Cd, Cu e Hg nas áreas estudadas.

\section{Soil trace elements in area of final solid urban residue disposals}

The trace elements can be present in the soil due to natural causes as well as by anthropogenic additions, being the last one worrying, bearing in mind that they cannot be degraded, being accumulated in the environment. Studies indicate high levels of trace elements in areas of final solid urban residue (SUR) disposals, but this does not always occur. This way, this project aimed to verify if the SUR disposal in the city of Pontes e Lacerda/MT, altered the local soil quality, analyzing the fertility attributes and the levels of trace elements $\mathrm{As}, \mathrm{Ba}, \mathrm{Co}, \mathrm{Cu}, \mathrm{Cr}, \mathrm{Cd}, \mathrm{Hg}, \mathrm{Mo}, \mathrm{Ni}, \mathrm{Pb}, \mathrm{Sb}$, Se and $\mathrm{Zn}$. Soil gatherings in the active final SUR disposals and in areas of native vegetation were made, totalizing 20 samples in the depth of 0-0,20 m. Alterations in chemical attributes were verified, especially with active and potential acidity, which were lower in the active area of final SUR disposal. The saturation per bases and K levels were more elevated in the active areas of final SUR disposals. The levels of As were higher in the native vegetation area. The levels of $\mathrm{Cd}, \mathrm{Hg}, \mathrm{Sb}$ and $\mathrm{As}$ were higher, including in the native vegetation area, this way, it is recommended that it be conducted new studies for a confirmation of these results and mainly to estabilish background values (VRQ) for local area. Levels were observed above the Value of Prevention (VP) established by CONAMA Resolution 420/2009 for the As, Cd, Cu and Hg in the studied areas, including the native vegetation areas.

Keywords: Nutrients; Heavy Metals; Waste Disposal; Contamination.

Topic: Engenharia Sanitária

Reviewed anonymously in the process of blind peer.
Received: 10/02/2018

Approved: 24/03/2018
Mayara Cristina Santos Marques

Instituto Federal de Mato Grosso, Brasil http://lattes.cnpq.br/3497252447430497

mayaracsmarques@gmail.com

\section{Maria Aparecida Pereira Pierangeli (iD)}

Universidade do Estado de Mato Grosso, Brasil

http://lattes.cnpq.br/4330841132184376

http://orcid.org/0000-0001-6453-080X

mapp@unemat.br

Geilson Xavier Rocha

Universidade do Estado de Mato Grosso, Brasil ttp://lattes.cnpq.br/1463904693777343

gxr.3agro@gmail.com

\section{Referencing this:}

MARQUES, M. C. S.; PIERANGELI, M. A. P.; ROCHA, G. X.. Elementostraço em solo de área de disposição final de resíduos sólidos urbanos. Revista Ibero Americana de Ciências Ambientais, v.9, n.3, p.161-173, 2018. DOI: http://doi.org/10.6008/CBPC2179-6858.2018.003.0014 


\section{INTRODUÇÃO}

As altas taxas de consumo de produtos diversos pelo ser humano geram uma crescente produção de resíduos sólidos urbanos (RSU), os quais estão entre os maiores problemas ambientais enfrentados pela humanidade (POLAZ et al., 2009). A quantidade de RSU produzidos, além do fator econômico, reflete os valores e hábitos de vida da população, determinantes do grau de disposição para a realização do consumo (GODECKE et al., 2012). Diante disso, a preocupação mundial em relação aos resíduos sólidos, em especial os domiciliares, tem aumentado com o gerenciamento inadequado e pela falta de áreas de disposição final (JACOBI et al., 2011).

No Brasil, o índice de destinação final adequada em 2014 foi de 58,4\%, porém, a quantidade de RSU destinada a locais inadequados totaliza, em média, 29.659 .170 toneladas ao ano $(41,6 \%)$, os quais seguiram para lixões ou aterros controlados. Estes últimos, por sua vez, do ponto de vista ambiental, pouco se diferenciam dos lixões, pois não possuem o conjunto de sistemas necessários para a proteção do meio ambiente e da saúde pública (ABELPRE, 2015).

Para completar, elementos-traço potencialmente tóxicos como chumbo $(\mathrm{Pb})$, mercúrio $(\mathrm{Hg})$, cádmio (Cd), arsênio (As), níquel ( $\mathrm{Ni}$ ) e cromo ( $\mathrm{Cr}$ ) estão presentes nos diversos tipos de resíduos levados para os lixões (MUÑOZ, 2002), podendo contaminar o solo e a agua gerando sérios problemas para a qualidade do ambiente e, consequentemente, dos seres vivos

Segundo Guilherme et al. (2005), os elementos-traço sempre serão onipresentes em solos, sedimentos e sistemas aquáticos e podem ser adicionados ao solo devido tanto a ações naturais (litogênica ou pedogênica), através do intemperismo de rochas e minerais e processos de formação do solo, emissões vulcânicas e outros; quanto por interferência humana, tais como aterros sanitários, atividades industriais e de mineração, aplicações de corretivos de solo, fertilizantes e defensivos agrícolas, entre outros.

Diferentemente de componentes da biosfera (ar, água e biota), os solos se apresentam não apenas como um dreno para contaminantes, mas também como tampões naturais que controlam o transporte de elementos químicos e outras substâncias para a atmosfera, hidrosfera e biota (KABATA-PENDIAS et al., 2001; GUILHERME et al., 2005). Entretanto, esta capacidade é limitada, podendo ocorrer alteração da sua qualidade, devido ao efeito cumulativo dos mesmos (CETESB, 2001). Assim, valores orientadores para referência de qualidade (VRQ) são importantes informações sobre os solos das localidades.

A variabilidade entre os tipos de elementos-traço predominantes nas áreas utilizadas como depósito de resíduos domiciliares pode estar associada à fase de degradação da matéria orgânica do resíduo, à quantidade e composição do material, às condições climáticas, à composição mineralógica do solo e à técnica analítica utilizada na determinação dos mesmos (FONTES et al., 2003; ZANELLO et al., 2009; ANICETO et al., 2012).

Dessa maneira, estudos têm indicado que áreas de aterros apresentam teores elevados de compostos orgânicos e elementos-traço (SISSINO et al., 1996), porém isso nem sempre se verifica conforme estudos de Alcântara et al. (2011) na área do Lixão de Cáceres/MT e de Oliveira et al. (2016) na área do Lixão 
de Humaitá/AM. Assim, este trabalho buscou verificar se a disposição de RSU na cidade de Pontes e Lacerda/MT alterou a qualidade do solo local, analisando-se os atributos de fertilidade e elementos-traço (As, Ba, Co, Cu, Cr, Cd, Hg, Mo, Ni, Pb, Sb, Se e Zn).

\section{MATERIAIS E MÉTODOS}

\section{Localização e Histórico da Área de Estudo}

O local amostrado foi a área de disposição final de resíduos sólidos urbanos (Figura 1) da cidade de Pontes e Lacerda/MT, localmente denominada de aterro controlado. A área, circundada de vegetação nativa, está localizada a aproximadamente $11 \mathrm{~km}$ do perímetro urbano, próxima às margens da Rodovia BR 174. No entorno, a aproximadamente $1 \mathrm{~km}$ localiza-se a Universidade do Estado de Mato Grosso - UNEMAT/Campus Pontes e Lacerda, o Presídio Municipal e a Mineração Aura Minerals.
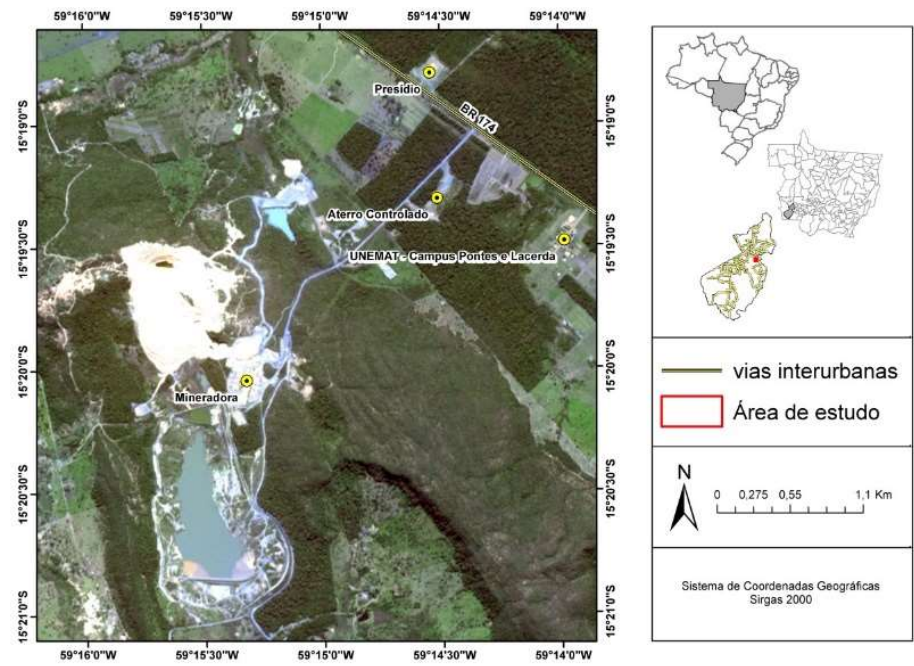

Figura 1: Localização do aterro controlado do município de Pontes e Lacerda/MT.

A região sudoeste de Mato Grosso, onde está localizado o município, é uma área de transição de três Biomas: Floresta Amazônica, Cerrado e Pantanal. O clima em Pontes e Lacerda, de acordo com a classificação de Köppen, é do tipo Aw, sendo a precipitação média anual de $1.275 \pm 154,0 \mathrm{~mm}$ e a temperatura média de 24,5 ํ․ Embora a área de disposição de RSU, em funcionamento há aproximadamente 15 anos, seja denominada de aterro controlado pelo poder público municipal, de acordo com suas condições de operação, a mesma mais se assemelha a um lixão, pois no local podem ser observados resíduos a céu aberto, presença de fogo, inexistência de controle de entrada e saída e, ainda, a presença de catadores no local.

Levando em consideração o valor per capita de coleta de RSU do estado de Mato Grosso que é de 0,853 kg/hab/dia (ABELPRE, 2015) e a população estimada do município, em 2016, de 43.538 habitantes, sendo $83,7 \%$ residente na área urbana (IBGE, 2016), atualmente, a área recebe, em média, 31 toneladas diárias de RSU. No entanto, considerando ainda que no local não existe um controle de entrada e saída de materiais, além dos resíduos sólidos domiciliares e comerciais provenientes da coleta municipal, resíduos de origens diversas podem ser levados até a área pelos seus próprios geradores, e, dessa maneira, a quantidade e a variabilidade de resíduos sólidos recebida na área pode ser maior. 


\section{Procedimento amostral}

Foram realizadas coletas em toda a área ativa de disposição final de RSU do município de Pontes e Lacerda/MT (Figura 3). As coletas foram realizadas em linha ('transecto linear') com pontos distanciados entre si em \pm 10 metros, totalizando 15 amostras, as quais foram coletadas na profundidade de 0-0,20 m. Ressalta-se que o denominado solo do depósito de resíduos sólidos é, na verdade, composto por uma mistura de materiais. Os RSU ao serem depositados e se decomporem são incorporados aos constituintes dos solos naturais passando, assim, a serem denominados de solos urbanos (ANICETO et al., 2012) ou Tecnossolos (WRB, 2006).

Como material de referência, foram coletadas amostras de solo em área de vegetação nativa, no entorno da área ativa de disposição final de RSU, na profundidade 0-0,20 m, totalizando 5 amostras. No total, foram coletadas 20 amostras para a realização de análises. Para subsidiar melhor entendimento do solo da área do aterro controlado foi aberta uma trincheira com medidas de 1,5 $\mathrm{m} \times 1,0 \mathrm{~m} \times 2,0 \mathrm{~m}$ (comprimento, largura e profundidade) na área de vegetação nativa, no entorno da área de disposição final de RSU, onde foi feita a descrição morfológica do solo conforme o IBGE de 2007. Posteriormente, o solo foi classificado conforme EMBRAPA (2013). Foram, ainda, realizadas a coleta de amostras de solo, por horizonte, para análises físicas e químicas.

\section{Procedimentos Analíticos}

Foram realizadas análises químicas para avaliação da fertilidade do solo (EMBRAPA, 2011): pH em água; P por colorimetria após extração com solução de Mehlich 1; K por espectrofotometria de emissão atômica após extração com solução de Mehlich 1; $\mathrm{Ca}^{2+} ; \mathrm{Mg}^{2+}$ por espectrofotometria de absorção atômica, após extração com solução de $\mathrm{KCl} 1 \mathrm{~mol} \mathrm{~L}^{-1}$; $\mathrm{Al}^{3+}$ extração por $\mathrm{KCl} 1$ mol.L-1 e determinação por titulometria; acidez potencial $(\mathrm{H}+\mathrm{Al})$ por acetato de cálcio $(0,5$ mol.L-1 $)$ e determinação por titulometria e pelo pH SMP; matéria orgânica após extração por solução de dicromato de sódio em ácido sulfúrico e determinação por titulometria com sulfato ferroso amoniacal. A textura do solo foi determinada pelo método da pipeta (EMBRAPA, 2011).

Os valores da soma de base (SB); capacidade de troca de cátions potencial (T); capacidade de troca de cátions efetiva (t); calculados em $\mathrm{cmol}_{\mathrm{c}} \mathrm{dm}^{-3}$ de TSFA foram obtidos de acordo com as seguintes expressões: $\mathrm{SB}=\mathrm{Ca}^{2+}+\mathrm{Mg}^{2+}+\mathrm{K}^{+}+\mathrm{Na}^{+} ; \mathrm{t}=\mathrm{SB}+\mathrm{Al}{ }^{3+} ; \mathrm{T}=\mathrm{SB}+(\mathrm{H}+\mathrm{Al}) ;$ a porcentagem de saturação por base $(V): V=(S B / T) \times 100 ;$ a porcentagem de saturação por alumínio trocável $(\mathrm{m}): \mathrm{m}=100 \times \mathrm{Al}^{3+} / \mathrm{t}$.

Nas amostras de solo foram realizadas, ainda, análises dos elementos-traço $\mathrm{As}, \mathrm{Ba}, \mathrm{Co}, \mathrm{Cu}, \mathrm{Cr}, \mathrm{Cd}, \mathrm{Hg}$, $\mathrm{Mo}, \mathrm{Ni}, \mathrm{Pb}$, Sb, Se e $\mathrm{Zn}$. O método analítico utilizado na extração dos teores totais de elementos-traço nas amostras de solos foi o USEPA SW-846 3051a (USEPA, 1998). As determinações de As, Ba, Co, Cu, Cr, Cd, $\mathrm{Hg}$, $\mathrm{Mo}, \mathrm{Ni}, \mathrm{Pb}$, Sb, Se e $\mathrm{Zn}$ foram realizadas por espectrometria de emissão óptica com plasma indutivamente acoplado com configuração axial (ICP-OES), conforme USEPA 6010C. Na determinação do Hg, utilizou-se a geração química de vapor acoplado a espectrometria de emissão óptica com plasma indutivamente acoplado 
com configuração axial (CVG-ICP OES) (USEPA, 1998; APHA, 2012). As análises foram realizadas pelo laboratório da Escola Superior Luiz de Queiroz - ESALQ da Universidade de São Paulo (USP), em Piracicaba/SP.

\section{Análise Estatística}

Foi realizado o teste de hipótese, por meio do Teste t não pareado (BEASLEY, 2004), adotando-se nível de significância de 5\%. As análises foram realizadas com o software R (R DEVELOPMENT CORE TEAM, 2016).

\section{RESULTADOS E DISCUSSÃO}

\section{Morfologia do Solo}

O solo da área de vegetação nativa, no entorno da área de disposição final de RSU, de acordo com os atributos morfológicos, físicos e químicos (Tabelas 1, 2 e 3) foi classificado como Argissolo Vermelho distrófico, pois conforme pode ser verificado com os dados da granulometria (Tabela 2) há um gradiente textural entre o A e o B igual a 1,9 e o horizonte Bt apresenta baixa saturação por bases ( $\mathrm{V}<50$ \%) (Tabela 3).

Tabela 1: Atributos morfológicos do solo da área de vegetação nativa no entorno da área de disposição final de RSU na cidade de Pontes e Lacerda/MT.

\begin{tabular}{|l|l|l|l|l|l|l|l|}
\hline Horiz. & Prof. $(\mathrm{cm})$ & Cor Munsell & Textura & Estrutura $^{1}$ & Consistência $^{2}$ & Transição $^{2}$ \\
\hline & & Seca & Úmida & & & & \\
\hline A & $5-20$ & 7,5 YR 4/6 & 2,5 YR 3/4 & Arenosa & fr., mpeq., bl. sub. & mac., fri., nplás. & Clara \\
\hline BA & $20-60$ & 5 YR 4/6 & 5 YR 4/4 & Média & fr., peq., bl. sub. & mac., fri., lig.plás. & Difusa \\
\hline B1 & $60-90$ & 5 YR 5/8 & 2,5 YR 4/6 & Média & mod., méd, bl. sub. & lig.dr., fri., lig.plás. & Difusa \\
\hline Bt & $90-140$ & 5 YR 5/8 & 2,5 YR 4/8 & Média & mod., peq., méd., gr., bl.sub. & mac., fri., lig.plás. & Difusa \\
\hline BC & $140+$ & 2,5 YR 5/8 & 2,5 YR 4/6 & Siltosa & fr., peq., méd., gr., bl. sub. & mac., fri., nplás. & Difusa \\
\hline
\end{tabular}

1fr.: fraca; mod.: moderada; peq.: pequena; mpeq.: muito pequena; méd.: média; gr.: grande; bl. sub.: blocos subangulares; ${ }^{2}$ mac.: macio; lig.dr.: ligeiramente duro; fri.: friável; nplás.: não plástico; lig. plás.: ligeiramente plástico.

Tabela 2: Atributos físicos do solo da área de vegetação nativa no entorno da área de disposição final de RSU na cidade de Pontes e Lacerda/MT.

\begin{tabular}{|l|l|l|l|l|l|l|}
\hline Horiz. & Prof. $(\mathrm{cm})$ & Areia $\left(\mathrm{g} \mathrm{kg}^{-1}\right)$ & Silte $\left(\mathrm{g} \mathrm{kg}^{-1}\right)$ & Argila $\left(\mathrm{g} \mathrm{kg}^{-1}\right)$ & $\mathrm{S} / \mathrm{A}^{1}$ & $\left.\mathrm{Ds}(\mathrm{mg} \mathrm{m})^{-3}\right)$ \\
\hline A & $5-20$ & 833 & 41 & 126 & 0,33 & 1,46 \\
\hline BA & $20-60$ & 736 & 37 & 227 & 0,16 & 1,43 \\
\hline B1 & $60-90$ & 724 & 50 & 226 & 0,22 & 1,54 \\
\hline Bt & $90-140$ & 698 & 52 & 250 & 0,21 & 1,56 \\
\hline BC & $140+$ & 711 & 37 & 251 & 0,15 & 1,52 \\
\hline
\end{tabular}

${ }^{1} \mathrm{~S} / \mathrm{A}=$ silte/argila; $\mathrm{Ds}=$ densidade do solo.

Tabela 3: Atributos químicos do solo da área de vegetação nativa no entorno da área de disposição final de RSU na cidade de Pontes e Lacerda/MT.

\begin{tabular}{|c|c|c|c|c|c|c|c|c|c|c|c|c|c|c|c|}
\hline Horiz. & Prof. & $\mathrm{pH} \mathrm{H} \mathrm{H}_{2} \mathrm{O}$ & $\mathrm{pH} \mathrm{CaCl}{ }_{2}$ & $\mathrm{MO}$ & $P$ & $\mathrm{~K}^{+}$ & $\mathrm{Ca}^{2+}$ & $\mathrm{Mg}^{2+}$ & $\mathrm{Al}^{3+}$ & $\mathrm{H}+\mathrm{Al}$ & $\mathrm{SB}^{1}$ & $t^{2}$ & $T^{3}$ & $\mathrm{~V}^{4}$ & $m^{5}$ \\
\hline & $\mathrm{cm}$ & & & $\mathrm{g} \mathrm{kg}^{-1}$ & \multicolumn{2}{|c|}{$\mathrm{mg} \mathrm{dm}^{-3}$} & \multicolumn{7}{|c|}{$\mathrm{cmolc} \mathrm{dm}^{-3}$} & $\%$ & \\
\hline A & $5-20$ & 5,5 & 4,3 & 15,7 & 1,7 & 36 & 0,7 & 1,0 & 0,7 & 3,6 & 1,8 & 2,49 & 5,4 & 33,0 & 28,0 \\
\hline $\mathrm{BA}$ & $20-60$ & 5,6 & 4,0 & 10,3 & 0,8 & 13 & 0,6 & 0,8 & 0,9 & 2,9 & 1,4 & 2,33 & 4,3 & 33,0 & 39,0 \\
\hline B1 & $60-90$ & 5,1 & 3,9 & 6,4 & 0,6 & 14 & 0,7 & 0,8 & 0,8 & 2,6 & 1,5 & 2,34 & 4,1 & 37,0 & 35,0 \\
\hline $\mathrm{Bt}$ & $90-140$ & 5,4 & 3,9 & 4,2 & 0,5 & 12 & 0,6 & 0,8 & 0,5 & 1,8 & 1,4 & 1,93 & 3,2 & 44,0 & 26,0 \\
\hline$B C$ & $140+$ & 5,4 & 4,2 & 3,2 & 0,4 & 11 & 0,5 & 0,8 & 0,4 & 1,9 & 1,3 & 1,73 & 3,2 & 41,0 & 23,0 \\
\hline
\end{tabular}

${ }^{1} \mathrm{SB}=$ soma de bases; ${ }^{2} \mathrm{t}=\mathrm{CTC}$ efetiva; ${ }^{3} \mathrm{~T}=\mathrm{CTC}$ potencial; ${ }^{4} \mathrm{~V}=$ saturação por bases; ${ }^{5} \mathrm{~m}=$ saturação por alumínio. 
A descrição morfológica do perfil do solo (Tabela 1) da área de vegetação nativa mostrou que esse apresenta sequência de horizontes A-BA-B1-Bt-BC, os quais se encontram sobre rochas metassedimentares (Formação Fortuna), segundo Fernandes et al. (2005). A cor úmida do solo variou de bruno avermelhado escuro no horizonte $A$ e vermelha no Bt e no BC.

\section{Granulometria do solo da área ativa de disposição final de RSU e da área de vegetação nativa no entorno}

Os resultados da análise granulométrica são apresentados na Tabela 4. Confirmando as análises da descrição morfológica do perfil do solo (Tabela 1 a 3) os solos da área de vegetação nativa e ativa de disposição final de RSU foram enquadrados como de textura média, conforme classificação do triângulo textural da Embrapa (2013), porém no limite com a textura arenosa.

Tabela 4: Teores médios das frações granulométricas na área ativa de disposição final de RSU e de vegetação nativa na cidade de Pontes e Lacerda/MT.

\begin{tabular}{|l|l|l|l|}
\hline \multirow{2}{*}{ Área } & Frações Granulométricas $\left(\mathrm{g} \mathrm{kg}^{-1}\right)$ & \multicolumn{2}{|l|}{} \\
\cline { 2 - 4 } & Areia & Silte & Argila \\
\hline Nativa & $826 \mathrm{a}$ & $31,6 \mathrm{a}$ & $142,4 \mathrm{a}$ \\
\hline Ativa & $723,2 \mathrm{~b}$ & $66,8 \mathrm{~b}$ & $209,9 \mathrm{~b}$ \\
\hline
\end{tabular}

(ab) médias seguidas da mesma letra minúscula em colunas não diferem pelo Teste t ao nível de 5\% de significância.

Maiores teores de argila na área ativa de disposição final de RSU, assim como os menores teores de areia, podem ser explicados pelo constante revolvimento do solo local para aterrar os RSU, onde materiais dos horizontes subsuperficiais, mais argilosos (Tabela 2) podem ser misturados com os da camada superficial. Alcântara et al. (2011), afirmam ainda que para teores de argila inferiores a 56 \% deve ser construído um sistema de impermeabilização e, de acordo com Oliveira et al. (2016), a natureza do solo da área representa inadequação para disposição de RSU.

Hamada et al. (2004) afirmaram a necessidade da compactação do solo local para as bases de aterros, resultando na redução da permeabilidade e garantindo um confinamento maior do chorume, sendo necessário para isso, solos com maiores teores de argila. Oliveira et al. (2004) afirmaram ainda que, os solos argilosos aparecem como uma alternativa barata e eficiente para impermeabilização superior, lateral e de fundo. Teores semelhantes das frações granulométricas também foram verificados por Oliveira et al. (2004) em um estudo realizado no aterro sanitário de Botucatu/SP, os quais concluíram que devido ao local do aterro ser formado por solo de textura média, com teores de $65 \%$ de areia e $27 \%$ de argila, havia maior possibilidade de lixiviação dos elementos-traço determinados ( $\mathrm{Cd}, \mathrm{Pb}, \mathrm{Cr}$, Ni e $\mathrm{Zn}$ ).

\section{Atributos de fertilidade do solo da área ativa de disposição final de RSU e da área de vegetação nativa no entorno}

Os resultados para os atributos químicos do solo relacionados à fertilidade para a área estudada são apresentados na Tabela 5. A acidez ativa $(\mathrm{pH})$ foi menor na área ativa de disposição final de RSU quando comparadas à nativa (Tabela 5). Menor valor de $\mathrm{pH}$ na área de vegetação nativa pode ser devido a mineralização da matéria orgânica e exsudatos ácidos liberados pelas raízes das plantas, fatores que podem também contribuir para aumentar a acidez do solo (OLIVEIRA et al., 2016). Os solos com alto teor de matéria 
orgânica tendem a ser mais ácidos, devido à formação dos ácidos orgânicos decorrentes da biodegradação de materiais orgânicos.

Tabela 5: Teores médios de atributos químicos do solo da área ativa de disposição final de RSU e de vegetação nativa na cidade de Pontes e Lacerda/MT.

\begin{tabular}{|c|c|c|}
\hline Atributos & Área Nativa & Área Ativa \\
\hline $\mathrm{pH} \mathrm{H}_{2} \mathrm{O}$ & $5,7 a$ & $6,45 b$ \\
\hline $\mathrm{pH} \mathrm{CaCl} 2$ & $4,64 a$ & $5,67 \mathrm{~b}$ \\
\hline $\mathrm{MO}\left(\mathrm{g} \mathrm{kg}^{-1}\right)$ & $22,96 a$ & $12,63 \mathrm{~b}$ \\
\hline $\mathrm{P}\left(\mathrm{mg} \mathrm{dm}^{-3}\right)$ & $5,4 a$ & $38,45 a$ \\
\hline $\mathrm{K}^{+}\left(\mathrm{mg} \mathrm{dm}^{-3}\right)$ & $68,4 a$ & $293,53 b$ \\
\hline $\mathrm{Ca}^{2+}\left(\mathrm{cmol}_{\mathrm{c}} \mathrm{dm}^{-3}\right)$ & $2,38 a$ & $3,29 a$ \\
\hline $\mathrm{Mg}^{2+}\left(\mathrm{cmol}_{\mathrm{c}} \mathrm{dm}^{-3}\right)$ & $1,06 a$ & $0,87 a$ \\
\hline $\mathrm{Al} 3+\left(\mathrm{cmol}_{\mathrm{c}} \mathrm{dm}^{-3}\right)$ & $0,2 \mathrm{a}$ & $0,03 a$ \\
\hline $\mathrm{H}+\mathrm{Al}\left(\mathrm{cmol}_{\mathrm{c}} \mathrm{dm}^{-3}\right)$ & $3,4 a$ & $1,07 b$ \\
\hline $\mathrm{SB}^{1}\left(\mathrm{cmol}_{\mathrm{c}} \mathrm{dm}^{-3}\right)$ & $3,6 a$ & $4,91 a$ \\
\hline $\mathrm{t}^{2}\left(\mathrm{cmol}_{\mathrm{c}} \mathrm{dm}^{-3}\right)$ & $3,81 a$ & $4,94 a$ \\
\hline $\mathrm{T}^{3}\left(\mathrm{cmol}_{\mathrm{c}} \mathrm{dm}^{-3}\right)$ & $7 a$ & $5,97 a$ \\
\hline $\mathrm{V}^{4}(\%)$ & $49 a$ & $80 \mathrm{~b}$ \\
\hline $\mathrm{m}^{5}(\%)$ & $7 a$ & $1 a$ \\
\hline
\end{tabular}

${ }^{1} \mathrm{SB}=$ soma de bases; ${ }^{2} \mathrm{t}=$ capacidade de troca catiônica efetiva; ${ }^{3} \mathrm{~T}=$ capacidade de troca catiônica potencial; ${ }^{4} \mathrm{~V}=$ saturação por bases; ${ }^{5} \mathrm{~m}$ = saturação por alumínio; (ab) médias seguidas da mesma letra minúscula em linhas não diferem pelo Teste $t$ ao nível de $5 \%$ de significância.

Ainda, devido à presença constante de fogo na área ativa de disposição final de RSU, há a possibilidade de as cinzas produzidas elevarem o pH na área, se comparados à área nativa. Um exemplo são os estudos recentes sobre o biocarvão ou biochar, que quando aplicado no solo, pode proporcionar aumento do pH (RONDON et al., 2006), ressaltando claro, as diferenças entre os materiais carbonizados e as cinzas.

Foram observadas diferenças estatísticas para a acidez potencial ( $\mathrm{H}+\mathrm{Al}$ ) (Tabela 5), tanto pelo método do acetato de cálcio quanto pelo método pH SMP, onde verificou-se um aumento da mesma na área de vegetação nativa, no entorno da área de disposição dos RSU. Tal fato está relacionado à maior acidez ativa na área de vegetação nativa, haja vista que nos solos tropicais quanto menor o pH, maior é a quantidade de $\mathrm{H}^{+}$retido nos colóides do solo. Quanto ao resultado na área ativa, Pavinato et al. (2008) explicaram que devido a complexação dos $\mathrm{H}^{+}$e $\mathrm{Al}^{3+}$ livres com compostos orgânicos aniônicos dos resíduos e do aumento da saturação da CTC do solo pelos $\mathrm{Ca}, \mathrm{Mg}$ e $\mathrm{K}$ adicionados, reduz-se a acidez potencial.

Não foram observadas diferenças estatísticas entre as áreas para os teores de $\mathrm{P}$, porém, conforme pode ser observado na Tabela 5, os teores de P encontram-se altos na área ativa de disposição de RSU (SOUZA et al., 2004). Vale salientar que os teores elevados de P disponível na área ativa de disposição final de RSU corroboram os resultados de Alcântara et al. (2011), Medeiros et al. (2008) e de Oliveira et al. (2016). Os últimos autores atribuem os altos teores de $\mathrm{P}$ à disposição de resíduos sem nenhum tratamento, em especial materiais orgânicos e detergentes. Porém, na área, os teores podem ser atribuídos ao lançamento de lodo proveniente da Estação de Tratamento de Esgoto - ETE do município.

Os teores de $\mathrm{K}$ foram elevados e estatisticamente maiores na área ativa de disposição de RSU (Tabela 5). Os resultados de $\mathrm{K} \mathrm{e} \mathrm{Ca}^{2+}$ da área nativa são semelhantes aos encontrados por Pierangeli et al. (2009) na mesma região, os autores destacam que esses teores podem ser em virtude de a área de estudo estar localizada na depressão do Guaporé, rica em rochas do grupo das sericitas e feldspatos potássicos e cálcicos. 
Ainda, segundo Fernandes et al. (2005) tal fato pode ser explicado devido à geologia regional, a qual compreende rochas sedimentares, granitos e granodioritos e rochas juvenis vulcânicas e plutônicas máficas.

Em relação à área ativa de disposição de RSU, houve um grande incremento na concentração de K, isso se deve provavelmente ao lançamento de resíduos sólidos, ricos em $\mathrm{K}$, sem nenhum controle na área. Ou, mais provavelmente os maiores teores de K na área ativa de disposição final de RSU podem ser, também, devido à lavagem ou decomposição do material orgânico depositado, a qual libera prontamente o K presente nos constituintes orgânicos, haja vista que o mesmo não faz parte de nenhuma molécula orgânica conhecida.

Para os atributos soma de bases (SB) e saturação por bases (V) (Tabela 5), os resultados vão de encontro aos obtidos por Oliveira et al. (2016) e Alcântara et al. (2011). Foram obtidos teores médios de saturação por base (V) na área de vegetação nativa e altos teores na área ativa de disposição dos RSU. Maiores teores de V na área ativa de deposição dos RSU se deve, em grande parte, aos altos teores de $\mathrm{K}$ (Tabela 5), o qual ocupa cerca de $13 \%$ da CTC total do solo.

Segundo Matias et al. (2012), alto V, nesse caso, é preocupante, visto que mostra que a quantidade de cátions saturando as cargas negativas é alta, dessa maneira, caso ocorra uma adição de elementos-traço, no caso da disposição de RSU, por exemplo, pode haver diminuição na retenção dos mesmos. Ainda, em estudo realizado por Zanello et al. (2009), os autores afirmam que altos teores de cátions bivalentes e trivalentes $\left(\mathrm{Ca}^{2+}, \mathrm{Mg}^{2+}\right.$ e $\left.\mathrm{Al}^{3+}\right)$ saturando as cargas negativas das argilas e da matéria orgânica dificulta a adsorção de elementos-traço adicionados ao solo via chorume.

Os valores de saturação por alumínio $(\mathrm{m})$ não foram estatisticamente diferentes entre as áreas e apresentaram-se baixos, corroborando estudo anterior (PIERANGELI et al., 2009) em solos da região. Esses baixos teores de $\mathrm{Al}^{3+}$ ocorrem devido ao $\mathrm{pH}$ do solo se encontrar acima de 5,5, valor em que o Al presente no solo se precipita como $\mathrm{Al}(\mathrm{OH})_{3}$, não estando presente na forma iônica, trocável.

Os teores de matéria orgânica foram estatisticamente maiores na área nativa (Tabela 5), em virtude de esta possuir vegetação natural, apesar da significativa parcela de resíduos sólidos urbanos composta por matéria orgânica, cerca de 50\% do total de resíduos sólidos (IPEA, 2012), dispostos diariamente na área ativa do aterro controlado. Esse resultado também foi encontrado por Alcântara et al. (2011) e Medeiros et al. (2008). Fato que pode ser explicado, pois a movimentação e o revolvimento do solo para escavação e aterramento provavelmente estão reduzindo a proteção física da matéria orgânica do solo (OLIVEIRA et al., 2004) e ainda, segundo Alcântara et al. (2011), o revolvimento do solo favorece a decomposição de matéria orgânica, onde os RSU são constituídos de materiais orgânicos de mais fácil decomposição (restos de alimentos, sobras de frutas e legumes, entre outros) (TEIXEIRA et al., 2000). Pode ser explicado também devido a camada de solo que é revolvido e misturada aos resíduos sólidos, durante o processo de aterramento dos mesmos, mobilizando as camadas subsuperficiais, mais pobres em matéria orgânica (Tabela 3). 
Teores de elementos-traço do solo da área ativa de disposição final de RSU e da área de vegetação nativa no entorno

Os teores de elementos-traço tanto da área ativa de disposição de RSU quanto da área de vegetação nativa são apresentados na Tabela 6. Comparando-se os teores médios encontrados no estudo em questão aos valores de referência de qualidade (VRQ) do estado de São Paulo (CETESB, 2014), observa-se que para os elementos-traço $\mathrm{Sb}, \mathrm{As}, \mathrm{Cd}, \mathrm{Cu}, \mathrm{Cr}$ e $\mathrm{Hg}$, tanto a área ativa de disposição final de resíduos sólidos urbanos quanto a área nativa apresentaram teores maiores que os VRQ do Estado de São Paulo (Tabela 6). O Pb apresentou teores maiores que os VRQ do estado de São Paulo na área nativa, no entorno do aterro controlado. Confrontando-se os valores dos VRQ propostos para o estado de MT e RO, segundo Santos et al. (2013), (Tabela 6), os teores dos elementos-traço Cd, Pb, Cu, Cr, Ni e Zn estão acima dos VRQ estipulados tanto na área nativa quanto na área ativa de disposição de resíduos sólidos urbanos.

Pierangeli et al. (2009) realizaram um trabalho na região a fim de verificar os teores naturais de elementos-traço no Sudoeste de MT e, comparando os resultados com os desse trabalho, os teores de Cd, $\mathrm{Pb}$ e Hg encontram-se mais elevados que aqueles, tanto na área ativa de disposição final de RSU quanto na área nativa (Tabela 6). Também, os teores de $\mathrm{As}, \mathrm{Cu}, \mathrm{Ni}$ na área de vegetação nativa e na área ativa de disposição de RSU, encontrados no presente trabalho, apresentaram-se superiores aos teores de elementotraço em áreas de vegetação nativa para o estado de Mato Grosso encontrados por Pierangeli et al. (2015) (Tabela 6).

Comparando-se aos Valores de Prevenção (VP), segundo a Resolução CONAMA 420/2009 (CONAMA, 2009), os teores de Sb, Cd, Cu e Hg apresentaram-se acima do estipulado na referida resolução, tanto para a área nativa, quanto para a área ativa de disposição final de RSU. Ainda, o teor do As encontrasse mais elevado que o VP para a área de vegetação nativa.

Tabela 6: Teores médios de elementos-traço do solo da área ativa de disposição final de RSU e de vegetação nativa na cidade de Pontes e Lacerda/MT.

\begin{tabular}{|c|c|c|c|c|c|c|c|}
\hline $\begin{array}{l}\text { Elemento } \\
\left(\mathrm{mg} \mathrm{kg}^{-1}\right)\end{array}$ & $\begin{array}{l}\text { Área } \\
\text { Nativa }\end{array}$ & $\begin{array}{l}\text { Área } \\
\text { Ativa }\end{array}$ & $\begin{array}{l}\text { VRQ } \\
\text { (CETESB, } \\
2014)\end{array}$ & $\begin{array}{l}\text { VRQ MT e } \\
\text { (SANTOS et al., } \\
2013)^{1}\end{array}$ & $\begin{array}{l}\text { Teores Naturais } \\
\text { Sudoeste MT } \\
\text { (PIERANGELI et } \\
\text { al., 2009) }\end{array}$ & $\begin{array}{l}\text { Teores Vegetação } \\
\text { Nativa MT } \\
\text { (PIERANGELI et } \\
\text { al., 2015) }\end{array}$ & $\begin{array}{l}\text { VP (CONAMA, } \\
2009)\end{array}$ \\
\hline Antimônio & $15,1 \mathrm{a}$ & $24,8 a$ & $<0,5$ & & & & 2 \\
\hline Arsênio & $21,1 a$ & $4,9 b$ & 3,5 & & 43,9 & 2,6 & 15 \\
\hline Bário & $17,2 a$ & $14,0 a$ & 75 & & & & 150 \\
\hline Cádmio & $2,6 a$ & $2,7 a$ & $<0,5$ & $<0,3$ & 0,45 & & 1,3 \\
\hline Chumbo & $21,0 a$ & $11,3 a$ & 17 & 9 & 11 & 19,2 & 72 \\
\hline Cobalto & $0,8 a$ & $1,0 a$ & 13 & 21,3 & & & 25 \\
\hline Cobre & $64,3 a$ & $65,8 a$ & 35 & 20,6 & & 7,9 & 60 \\
\hline Cromo & $58,2 a$ & $58,0 a$ & 40 & 44,8 & & 76,4 & 75 \\
\hline Mercúrio & $7,2 a$ & $11,3 a$ & 0,05 & & 0,14 & & 0,5 \\
\hline Molibdênio & $0,1 a$ & $0,1 a$ & $<4$ & & & & 30 \\
\hline Níquel & $5,7 a$ & $5,9 a$ & 13 & 2,1 & & 2,1 & 30 \\
\hline Selênio & $0,1 a$ & $0,1 a$ & 0,25 & & & & 5 \\
\hline Zinco & $8,5 a$ & $25,6 a$ & 60 & 3 & & 8,2 & 300 \\
\hline
\end{tabular}

(ab) médias seguidas da mesma letra minúscula em linhas não diferem pelo Teste t ao nível de $5 \%$ de significância; ${ }^{1}$ Percentil 75.

Destacam-se ainda os teores encontrados para o Hg (Tabela 6). O teor deste, ainda, aumenta na área ativa de disposição final de resíduos sólidos urbanos. O município de Pontes e Lacerda destaca-se no cenário 
nacional na produção de ouro e tal fato pode explicar os altos índices de $\mathrm{Hg}$ encontrados na região, em especial na área ativa de disposição final de resíduos sólidos, visto que próxima à área existe uma mineração, a qual durante muitos anos funcionou como um garimpo a céu aberto, utilizando o mercúrio para amalgamação. Os teores nas áreas estudadas podem estar relacionados ao transporte atmosférico do $\mathrm{Hg}$, por meio das chuvas, a partir da evaporação por meio das superfícies terrestres e aquáticas, onde o solo se torna um reservatório (CLARKSON, 2002). Outros estudos em solos amazônicos têm relatado altos teores de Hg nos solos (OLIVEIRA et al., 2007).

Com relação aos teores encontrados de Hg, Lázaro et al. (2013) afirmam que algumas áreas são mais vulneráveis à contaminação ambiental por $\mathrm{Hg}$, como por exemplo o Pantanal na época de inundação. Outro exemplo está na região Amazônica, onde segundo Roulet et al. (2000), ao realizar um estudo no Vale do Rio Tapajós, as comunidades perifíticas são uma das principais fontes de $\mathrm{MeHg}$, formados a partir do $\mathrm{Hg}$.

Os teores encontrados para o As, que se destaca no cenário mundial devido a sua toxicidade, são estatisticamente maiores na área de vegetação nativa, sem disposição de resíduos sólidos urbanos (Tabela 6). Esses altos teores de As também foram reportados por Pierangeli et al. (2009) em estudo realizado na mesma região, fato que, pode estar relacionado à litologia regional (FERNANDES et al., 2005), a qual apresenta rochas com altos teores de As $\left(>3.800 \mathrm{mg} \mathrm{kg}^{-1}\right)$.

Os teores de Cd também foram teores elevados. Para McBride (1994) teores de Cd no solo acima de $0,5 \mathrm{mg} \mathrm{kg}^{-1}$ já é indicativo de contaminação, pois esse elemento é altamente tóxico para animais e plantas. Porém, não foram observadas diferenças estatísticas entre as áreas (Tabela 6), fato que descarta a possibilidade de dos altos teores serem decorrentes de contaminação.

Não foram observadas diferenças entre as áreas com relação ao Ni (Tabela 6), este que apresentou ligeiro aumento na área ativa em relação à área nativa, que pode ser decorrente do lançamento de resíduos sólidos que contenham esse elemento, como pilhas e baterias. Já a concentração de Cu pode ser explicada devido a geologia local, devido a presença de rochas máficas (FERNANDES et al., 2005), estas que apresentam altos teores de Cu (ALLOWAY, 1990).

Os teores de $\mathrm{Pb}$ encontrados no solo da área de vegetação nativa são semelhantes aos obtidos por Pierangeli et al. (2015). Tal fato pode ser proveniente da baixa mobilidade desse metal no perfil do solo, da sua elevada adsorção na fase sólida (inorgânica e orgânica) e sua capacidade de se acumular na camada superficial do solo (FERNANDES et al., 2011). Pierangeli et al. (2001) relataram uma maior contribuição das rochas máficas para elevação dos teores de $\mathrm{Pb}$ nos solos estudados, a qual é encontrada na região segundo Fernandes et al. (2005), onde os teores obtidos na área nativa vão ao encontro do estudo realizado por Pierangeli et al. (2015) (Tabela 6).

Os teores de $\mathrm{Zn}$ da área de vegetação nativa foram semelhantes aos encontrados por Pierangeli et al. (2015) em áreas de vegetação nativa do estado de Mato Grosso (Tabela 6). Embora as concentrações tenham sido superiores na área ativa de disposição de RSU, não houve diferenças estatísticas entre as áreas (Tabela 6). Quanto aos teores encontrados de Sb, Kabata-Pendias et al. (2001) afirmam que podem estar associados com depósitos de minério não-ferrosos, e também é considerado um poluente em ambientes 
industriais. Usos modernos de Sb incluem: como catalisador na fabricação de PET, como revestimento de cabo, munições e rolamentos, ligas metálicas para revestimento de baterias, lâminas, tubos e moldes metálicos (SMICHOWSKI, 2008).

\section{CONCLUSÕES}

Foram verificadas alterações nos atributos químicos do solo na área ativa de disposição de resíduos, em especial, acidez ativa e potencial, na saturação por bases e K. Os teores dos elementos $\mathrm{Ba}, \mathrm{Co}, \mathrm{Cu}, \mathrm{Cr}, \mathrm{Cd}$, $\mathrm{Hg}, \mathrm{Mo}, \mathrm{Ni}, \mathrm{Pb}, \mathrm{Sb}$, Se e Zn foram iguais entre a área ativa de disposição final dos resíduos sólidos urbanos e vegetação nativa no entorno. Os teores de As foram mais elevados na área de vegetação nativa.

Os teores de elementos-traço encontrados, por exemplo, $\mathrm{As}, \mathrm{Cu}$ e $\mathrm{Pb}$, foram atribuídos ao material de origem da região. Os teores de $\mathrm{Hg}$ foram elevados, inclusive na área de vegetação nativa, sendo assim, recomenda-se a realização de novos estudos para a confirmação dos resultados e estabelecimento de valores realistas de teores nativos.

Foram observados teores acima dos Valores de Prevenção da Resolução CONAMA 420/2009 para o As, $\mathrm{Cd}, \mathrm{Cu}$ e $\mathrm{Hg}$ nas áreas estudadas, inclusive na área nativa. Assim, tal fato, subsidia a necessidade de obtenção de Valores de Referência de Qualidade para o estado de Mato Grosso. Devido à natureza do solo da área, com grandes teores de areia, que conferem maior permeabilidade ao solo, mesmo sendo profundo, recomenda-se a impermeabilização da área para o lançamento dos RSU.

\section{REFERÊNCIAS}

ABELPRE. Associação Brasileira de Empresas de Limpeza Pública e Resíduos Especiais. Panorama de Resíduos Sólidos no Brasil- 2014. São Paulo: Abrelpe, 2015.

ALCÂNTARA, A. J. O.; PIERANGELI, M. A. P.; SOUZA, C. A.; SOUZA, J. B.. Teores de $\mathrm{As}, \mathrm{Cd}, \mathrm{Pb}, \mathrm{Cr}$ e $\mathrm{Ni}$ e atributos de fertilidade de Argissolo Amarelo distrófico usado como lixão no município de Cáceres, estado de Mato Grosso. Revista Brasileira de Geociências, v.41, n.3, p.539-548, 2011.

ALLOWAY, B. J.. Heavy metals in soils. Blackie Academic \& Professional. New York: John Wiley \& Sons, 1990.

ANICETO, K. C. P.; HORBE, A. M. C.. Solos urbanos formados pelo acúmulo de resíduos em Manaus, Amazonas, Brasil, Acta Amazonica, v.42, n.1, p.135-148, 2012. DOI: http://dx.doi.org/10.1590/S0044-59672012000100016

BEASLEY, C. R.. Bioestatística usando o R: Apostila de exemplos para o biólogo. Bragança: Universidade Federal do Pará, 2004.

CELERE, M. S.; OLIVEIRA, A. S.; TREVILATO, T. M. B.; SEGURAMUÑOZ, S. I.. Metais presentes no chorume coletado no aterro sanitário de Ribeirão Preto, São Paulo, Brasil, e sua relevância para saúde pública. Cad. Saúde Pública, Rio de Janeiro, v.23, n.4, p.939-947, 2007. DOI: http://dx.doi.org/10.1590/s0102-311X2007000400021

CETESB. Companhia de Tecnologia de Saneamento Ambiental. Relatório de estabelecimento de Valores
Orientadores para Solos e Águas Subterrâneas no Estado de São Paulo. 2001.

CETESB. Companhia de Tecnologia de Saneamento Ambiental. Relatório de estabelecimento de Valores Orientadores para Solos e Águas Subterrâneas no Estado de São Paulo. São Paulo, 2014.

CLARKSON, T. W.. The Three Modern Faces of Mercury. Environmental Health Perspectives, v.111, p.11-23, 2002.

CONAMA. Conselho Nacional do Meio Ambiente. Resolução no 420/2009: Dispõe sobre critérios e valores orientadores de qualidade do solo quanto à presença de substâncias químicas e estabelece diretrizes para o gerenciamento ambiental de áreas contaminadas por essas substâncias em decorrência de atividades antrópicas. Brasília: DOU, 2009.

EMBRAPA. Empresa Brasileira de Pesquisa Agropecuária. Manual e métodos de análise de solos. 2 ed. Rio de Janeiro: Embrapa Solos, 2011.

EMBRAPA. Empresa Brasileira de Pesquisa Agropecuária. Sistema brasileiro de classificação de solos. 3 ed. Brasília, 2013.

FERNANDES, C. J.; RUIZ, A. S.; PINHO, F. E. C.; KUYUMJIAN, R. M.. Compartimentação tectônica da deformação na Faixa Móvel Aguapeí, sudoeste do Cráton Amazônico, e as mineralizações auríferas associadas. Revista Brasileira de Geociências, v.35, n.1, p.1-12, 2005. 
FONTES, M. P. F.; GOMES, P. C.. Simultaneous competitive adsorption of heavy metals by the minerals matrix of tropical soils. Applied Geochemistry, n.18, p.795-804, 2003.

GODECKE, M. V.; NAIME, R. H.; FIGUEIREDO, J. A. S.. O consumismo e a geração de resíduos sólidos urbanos no Brasil. Rev. Elet. em Gestão, Educação e Tecnologia Ambiental, v.8, n.8, p.1700-1712, 2012. DOI: http://dx.doi.org/10.5902/223611706380

GUILHERME, L. R. G.; MARQUES, J. J.; PIERANGELI, M. A. P.; ZULIANE, D. Q.; CAMPOS, M. L.; MARCHI, G.. ElementosTraço em solos e sistemas aquáticos. In: TORRADO-VIDAL, P.; ALLEONI, L. R. F.; COOPER, M.; SILVA, A. P.. Tópicos em Ciência do Solo. 4 ed. 2005, p.345-390.

HAMADA, J.; CALÇAS, D. A. N. Q. P.; GIACHETI, H. L.. Influência da compactação de um solo arenoso na infiltração e retenção de carga orgânica de chorume. Eng. Sanit. Ambient., Rio de Janeiro, v.9, n.3, p.180-186, 2004.

IBGE. Instituto Brasileiro de Geografia e Estatística. Cidades. 2016.

IPEA. Instituto de Pesquisa Econômica Aplicada. Diagnóstico dos Resíduos Sólidos Urbanos. Brasília: IPEA, 2012.

JACOBI, P. R.; BENSEN, G. R.. Gestão de resíduos sólidos em São Paulo: desafios da sustentabilidade. Rev. Estud. Av, São Paulo, v.25, n.71, 2011, p.135-158. DOI: http://dx.doi.org/10.1590/S0103-40142011000100010

KABATA-PENDIAS, A.; PENDIAS, H.. Trace elements in soils and plants. 3 ed. Boca Raton: CRC Press, 2001.

LÁZARO, W. L.; GUIMARÃES, J. R.; IGNÁCIO, A. R. A.; SILVA, C. J.; DÍEZ, S.. Cyanobacteria enhance methylmercury production: A hypothesis tested in the periphyton of two lakes in the Pantanal floodplain, Brazil. Science of the Total Environment, v.256-257, p.231-238, 2013. DOI: http://dx.doi.org/10.1016/j.scitotenv.2013.03.022

MATIAS, D. N.; COSTA, W.. Estudo químico de alguns pontos do solo superficial do aterro controlado do Botuquara.

Ambiência, v.8, n.1, p.85-99, 2012.

MCBRIDE, M. D.. Environmental chemistry of soils. New York: Oxford University, 1994.

MEDEIROS, G. A.; REIS, F. A. G. V.; SIMONETTI, F. D.; BATISTA, G.; MONTEIRO, T.; CAMARGO, V.; SANTOS, L. F. S.; RIBEIRO, L. F. M.. Diagnóstico da qualidade da água e do solo no lixão de Engenheiro Coelho, no estado de São Paulo. Engenharia Ambiental, v.5, n.2, p.169-186, 2008.

MUÑOZ, S. I. S.. Impacto ambiental na área do aterro sanitário e incinerador de resíduos sólidos de Ribeirão Preto/SP: avaliação dos níveis de metais pesados. Tese (Doutorado) - Universidade de São Paulo, São Paulo.

OLIVEIRA, F. S. J.; JUCÁ, F. T. J.. Acúmulo de metais pesados e capacidade de impermeabilização do solo imediatamente abaixo de uma célula de um aterro de resíduos sólidos. Eng Sanit Ambient., v.9, n.3, p.211-217, 2004.
OLIVEIRA, L. C.; SERUDO, R. L.; BOTERO, W. G.; MENDONÇA, A. G. R.; SANTOS, A.; ROCHA, J. C.; NETO, F. S. C.. Distribuição de mercúrio em diferentes solos da bacia do Médio Rio Negro-AM: influência da matéria orgânica no ciclo biogeoquímico do mercúrio. Química Nova, v.30, n.2, p.274280, 2007.

OLIVEIRA, B. O. S.; TUCCI, C. A. F.; NEVES JR, A. F.; SANTOS, A. A.. Avaliação dos solos e das águas nas áreas de influência de disposição de resíduos sólidos urbanos de Humaitá, Amazonas. Eng. Sanit. Ambient., Rio de Janeiro, v.21, n.3, p.593-601, 2016. DOI: http://dx.doi.org/10.1590/S141341522016133274

PAVINATO, P. S.; ROSOLEM, C. A.. Disponibilidade de nutrientes no solo - decomposição e liberação de compostos orgânicos de resíduos vegetais. R. Bras. Ci. Solo, v.32, n.3, p.911-920, 2008. DOI: http://dx.doi.org/10.1590/S010006832008000300001

PIERANGELI, M. A. P.; GUILHERME, L. R. G.; CURI, N.; SILVA, M. L. N.; OLIVEIRA, L. R.; LIMA, J. M.. Teor total e capacidade máxima de adsorção de chumbo em latossolos brasileiros. $\mathbf{R}$. Bras. Ci. Solo, v.25, n.2, p.279-288, 2001.

PIERANGELI, M. A. P.; GUILHERME, L. R. G.; CURI, N.; SILVA, M. L. N.; LIMA, J. M.; COSTA, E. T. S.. Efeito do pH na adsorção e dessorção de cádmio em Latossolos brasileiros. Rev. Bras. Ciênc. Solo, v.29, n.4, p.523-532, 2005. DOI: http://dx.doi.org/10.1590/S0100-06832005000400005

PIERANGELI, M. A. P.; EGUCHI, E. S.; RUPPIN, R. F.; COSTA, R. B. F.; VIEIRA, D. F.. Teores de As, $\mathrm{Pb}, \mathrm{Cd}$ e Hg e fertilidade de solos da região do Vale do Alto Guaporé, sudoeste do Estado de Mato Grosso. Acta Amazonica, v.39, n.1, p.61-69, 2009.

PIERANGELI, M. A. P.; CARVALHO, G. S.; MARQUES, J. J.; CARVALHO, C. A.; PIERANGELI, L. M. P.; GUILHERME, L. R. G.. Elementos-traço em áreas de vegetação nativa e agricultura intensiva no Estado de Mato Grosso, determinados por Fluorescência de Raios-X por Reflexão Total. R. Bras. Ci. Solo, v.39, n.4, p.1048-1057, 2015. DOI: http://dx.doi.org/10.1590/01000683rbcs20140373

POLAZ, C. N. M.; TEIXEIRA, B. A. N.. Indicadores de sustentabilidade para a gestão municipal de resíduos sólidos urbanos: um estudo para São Carlos/SP. Eng. Sanit. Ambient., Rio de Janeiro, v.14, n.3, p.411-420, 2009. DOI: http://dx.doi.org/10.1590/S1413-41522009000300015

R DEVELOPMENT CORE TEAM. R: a language and environment for statistical computing. Vienna: R Foundation for Statistical Computing, 2016

RONDON, M. A.; LEHMANN, J.; RAMÍREZ, J.; HURTADO, M.. Biological nitrogen fixation by common beans (Phaseolus vulgaris L.) increases with bio-char additions. Biology Fertility Soils, v.43, p.699-708, 2006. DOI: http://dx.doi.org/10.1007/s00374-006-0152-z

ROULET, M.; LUCOTTE, M.; GUIMARÃES, J. R. D.; RHEAULT, I.. Methylmercury in water, seston, and epiphyton of an Amazonian river and its floodplain, Tapajós River, Brazil. Science Total Environmental, v.261, p.43-59, 2000.

SANTOS, S. N.; ALLEONI, L. F. R.. Reference values for heavy metals in soils of the Brazilian agricultural frontier in 
Southwestern Amazônia. Environ Monit Assess, v.185, n.7, p.5737-5748, 2013. DOI: http://dx.doi.org/10.1590/S0100$\underline{06832014000300035}$

SISSINO, C. L. S.; MOREIRA, J. C.. Avaliação da contaminação e poluição ambiental na área de influência do aterro controlado do Morro do Céu, Niterói, Brasil. Cadernos de Saúde Pública, Rio de Janeiro, v.12, n.4, p.515-523, 1996.

SMICHOWSKI, P.. Antimony in the environment as a global pollutant: a review on analytical methodologies for its determination in atmospheric aerosols. Talanta, v.1, n.75, p.2-14, 2008. DOI:

http://dx.doi.org/10.1016/j.talanta.2007.11.005
SOUZA, D. M. G.; LOBATO, E.. Cerrado: correção do solo e adubação. 2 ed. Brasília: Embrapa Cerrados, 2004.

TEIXEIRA, L. B.; OLIVEIRA, R. F.; FURLAN JUNIOR, J.; CRUZ, E. S.; GERMANO, V. L. C.. Compostagem de lixo orgânico urbano no município de Barcarena/Pará. Belém: Embrapa Amazônia Oriental, 2000.

ZANELLO, S.; MELO, V. F.; WOWK, G. I. T. H.. Mineralogia e teores de cromo, níquel, cobre, zinco e chumbo nos solos no entorno do aterro sanitário da Caximba em Curitiba/PR. Scientia Agraria, Curitiba, v.10, n.1, p.51-60, 2009.

WRB. World reference base for soil resources. World soil resources report. 2 ed. FAO, 2007.

A CBPC - Companhia Brasileira de Produção Científica (CNPJ: 11.221.422/0001-03) detém os direitos materiais desta publicação. Os direitos referem-se à publicação do trabalho em qualquer parte do mundo, incluindo os direitos às renovações, expansões e disseminações da contribuição, bem como outros direitos subsidiários. Todos os trabalhos publicados eletronicamente poderão posteriormente ser publicados em coletâneas impressas sob coordenação da Sustenere Publishing, da Companhia Brasileira de Produção Científica e seus parceiros autorizados. Os (as) autores (as) preservam os direitos autorais, mas não têm permissão para a publicação da contribuição em outro meio, impresso ou digital, em português ou em tradução. 